\title{
Indirect Field-oriented Control of Induction Motors is Robustly Globally Stable
}

\author{
PAUL A. S. DE WIT, ${ }^{\dagger}$ ROMEO ORTEGA ${ }^{\ddagger}$ and IVEN MAREELS $\$$
}

\begin{abstract}
Robustness to uncertain parameters of this popular induction motor controller is investigated. Conditions for local and global asymptotic stability as well as boundedness of all signals are derived.
\end{abstract}

Key Words - Induction motors; nonlinear control; stability analysis.

\begin{abstract}
Field orientation, in one of its many forms, is an established control method for high dynamic performance $\mathrm{AC}$ drives. In particular, for induction motors, indirect fieldoriented control is a simple and highly reliable scheme which has become the de facto industry standard. In spite of its widespread popularity no rigorous stability proof for this controller was available in the literature. In a recent paper (Ortega et al., 1995) [Ortega, R., D. Taoutaou, R. Rabinovici and J. P. Vilain (1995). On field oriented and passivity-based control of induction motors: downward compatibility. In Proc. IFAC NOLCOS Conf., Tahoe City, CA.] we have shown that, in speed regulation tasks with constant load torque and current-fed machines, indirect field-oriented control is globally asymptotically stable provided the motor rotor resistance is exactly known. It is well known that this parameter is subject to significant changes during the machine operation, hence the question of the robustness of this stability result remained to be established. In this paper we provide some answers to this question. First, we use basic input-output theory to derive sufficient conditions on the motor and controller parameters for global boundedness of all solutions. Then, we give necessary and sufficient conditions for the uniqueness of the equilibrium point of the (nonlinear) closed loop, which interestingly enough allows for a $200 \%$ error in the rotor resistance estimate. Finally, we give conditions on the motor and controller parameters, and the speed and rotor flux norm reference values that insure (global or local) asymptotic stability or instability of the equilibrium. This analysis is based on a nonlinear change of coordinates and classical Lyapunov stability theory. Copyright (C)1996 Elsevier Science Ltd.
\end{abstract}

Received 14 February 1995; revised 7 February 1996; received in final form 23 March 1996. This paper was not presented at any IFAC meeting. This paper was recommended for publication in revised form by Associate Editor Darren Dawson under the direction of Editor Yaman Arkun. Corresponding author Dr Paul A. S. de Wit. Tel. +31 53489 2520; Fax +31 53489 3631; E-mail p.a.s.dewit@wb.utwente.nl.

$\dagger$ Faculty of Mechanical Engineering, University of Twente, PO Box 217, 7500 AE Enschede, The Netherlands.

₹ URA C.N.R.S., Université de Technologie de Compiègne, 817, BP 649, 60206 Compiègne, France (rortega@hds.univcompiegne.fr).

$\$$ Department of Systems Engineering, Australian National University, Canberra, ACT 0200, Australia.

\section{PROBLEM FORMULATION}

In this paper we carry out the stability analysis of an indirect field oriented controller (FOC) that regulates the velocity and the rotor flux norm of a current-fed induction motor in the presence of an unknown constant load torque and rotor resistance uncertainty. For further details and motivation of induction motors and FOC the reader is refered to Bose (1986), Leonhard (1985), in the electrical machines literature, and to Bodson et al. (1994), Ortega et al. (1995), Taylor (1994) in the control journals.

The dynamic model of the current-fed induction motor in its simplest formulation expresses the rotor flux and the stator currents in a reference frame rotating at the rotor angular speed. *

$$
\begin{aligned}
& \dot{x}=-\frac{1}{T_{\mathrm{r}}} x+\frac{L_{m}}{T_{\mathrm{r}}} u=-R_{\mathrm{r}} x+R_{\mathrm{r}} u, \\
& \dot{y}=\frac{1}{M}\left(\tau-\tau_{L}\right)=\tau-\tau_{L}, \\
& \tau=u^{\mathrm{T}} L_{m} J x=u^{\mathrm{T}} J x,
\end{aligned}
$$

where

$$
\begin{array}{r}
x=\left[\begin{array}{l}
x_{1} \\
x_{2}
\end{array}\right] \in \mathbb{R}^{2}-\text { rotor flux vector, } \\
\begin{array}{r}
u=\left[\begin{array}{l}
u_{1} \\
u_{2}
\end{array}\right] \in \mathbb{R}^{2}-\text { stator currents, } \\
y-\text { rotor velocity, } \\
\tau-\text { generated torque, } \\
T_{\mathrm{r}}=\frac{L_{\mathrm{r}}}{R_{\mathrm{r}}}-\text { rotor time constant, }
\end{array}
\end{array}
$$

\footnotetext{
* See Bodson et al. (1994) for the derivation of this model from the classical textbook models.
} 


$$
\begin{aligned}
& R_{\mathrm{r}}>0 \text { - rotor resistance, } \\
& L_{\mathrm{r}}=1-\text { rotor self-inductance, } \\
& L_{m}=1 \text { - mutual inductance, } \\
& M=1 \text { - rotor inertia, } \\
& \tau_{L}-\text { load torque } \\
& \text { and } J=\left[\begin{array}{rr}
0 & -1 \\
1 & 0
\end{array}\right] .
\end{aligned}
$$

To simplify the expressions below, and without loss of generality for the purposes of this study, all motor parameters have been set to unity exccpt the rotor resistance and the load torque, which are assumed constant but unknown.

In indirect FOC, the stator currents are chosen as (Ortega et al., 1996; Ortega et al., 1995)

$$
u=\mathrm{e}^{J \rho_{\mathrm{d}}}\left[\begin{array}{c}
\beta \\
\frac{\tau_{\mathrm{d}}}{\beta}
\end{array}\right]
$$

Here $\beta>0$ is the constant desired value of the rotor flux norm. The angle of the desired rotor flux $\rho_{\mathrm{d}}$ is given by

$$
\dot{\rho}_{\mathrm{d}}=\frac{\hat{R}_{\mathrm{r}}}{\beta^{2}} \tau_{\mathrm{d}}
$$

with $\hat{R}_{\mathrm{r}}>0$ the constant estimated rotor resistance. The desired torque is $\tau_{\mathrm{d}}$. In velocity regulation applications, the latter is typically defined via a PI velocity loop as

$$
\tau_{\mathrm{d}}=-\left(K_{P}+\frac{K_{I}}{p}\right)\left(y-y_{\mathrm{d}}\right),
$$

where $y_{\mathrm{d}}$ is the desired velocity, which we assume constant, $p=\frac{\mathrm{d}}{\mathrm{d} t}$ is the derivative operator, and $K_{P}, K_{I} \geq 0$ are the PI tuning gains.

In summary, the closed loop is described by a fourth-order nonlinear autonomous system, whose block diagram description is given in Fig. 1 .

The problems we solve in this paper are the following:

\section{Stability analysis of indirect FOC.}

Given the motor model (1), (2), (3) in closed loop with the indirect FOC (4), (5), (6), find sufficient conditions on the motor parameters $R_{\mathrm{r}}, \tau_{L}$, the controller parameters $\hat{R}_{\mathrm{r}}, K_{P}, K_{I}$, and the reference values $y_{\mathrm{d}}, \beta$ such that:

1. All solutions of the system are globally bounded.

2. The system is (globally or locally) asymptotically stable. That is, such that

$$
\lim _{t \rightarrow \infty} y=y_{\mathrm{d}}, \quad \lim _{t \rightarrow \infty}|x|=\bar{\beta},
$$

where $|\cdot|$ is the Euclidean norm, and $\bar{\beta}$ denotes a constant value for the rotor flux norm; $\bar{\beta}$ does not have to equal $\beta$.
3. The system has unstable equilibria.

\section{Discussion}

- We analyze a current-fed induction motor. which means we assume that the stator currents $u_{1}(l), u_{2}(t)$ are equal to possibly discontinuous setpoints calculated by the controller. In real motors, stator currents must be continuous so that they cannot exactly follow discontinuous setpoints. However, the stator currents can follow the setpoints well if the leakage inductances are sufficiently small and the voltage source can supply a sufficiently high voltage (Hughes et al., 1993).

- Setting all parameters except the rotor resistance to unity causes no loss of generality, for two reasons. First, setting rotor inertia and mutual inductance to unity changes only the loop gain by a factor, which can be compensated for by scaling $K_{P}, K_{I}$. Second, the unknown parameter of importance to indirect field-oriented control is the rotor time constant, which is a function of both rotor resistance and rotor self-inductance. Therefore, the effect of an unknown rotor time constant can be investigated by considering the effect of an unknown rotor resistance only. To generalize the uniqueness and stability conditions derived in this paper, replace $R_{\mathrm{r}}$ by $R_{\mathrm{r}} / L_{\mathrm{r}}$ and $\hat{R}_{\mathrm{r}}$ by $\hat{R}_{\mathrm{r}} / \hat{L}_{\mathrm{r}}$.

- It is important to underscore the fact that $x$ is a vector quantity. This model should not be confused with the machine model in decoupling control, e.g. (7.75), (7.82), (2.78) of Bose (1986), which describes the asymptotic behaviour of the motor in closed loop with an ideal direct FOC.

- Interestingly, indirect FOC of a current-fed induction motor is obtained as a particular case of the passivity-based controller proposed in Ortega et al. (1996) when the stator current dynamics are neglected. If these dynamics are to be taken into account the full controller of Ortega et al. (1996) (see also Ortega et al. (1995) and Ortega and Espinosa (1993)) is required for global asymptotic stability (GAS). To the best of our knowledge, this practically appealing 'downward compatibility' property of the passivity-based control is absent in the controllers based on feedback linearization (e.g. Kim et al., 1990; Marino et al., 1993; Chiasson, 1993; Kanellakopoulos et al., 1992).

- As discussed in Ortega et al. (1995) one way of explaining the rationale underlying indirect FOC is to compare it with direct FOC, which is described by 


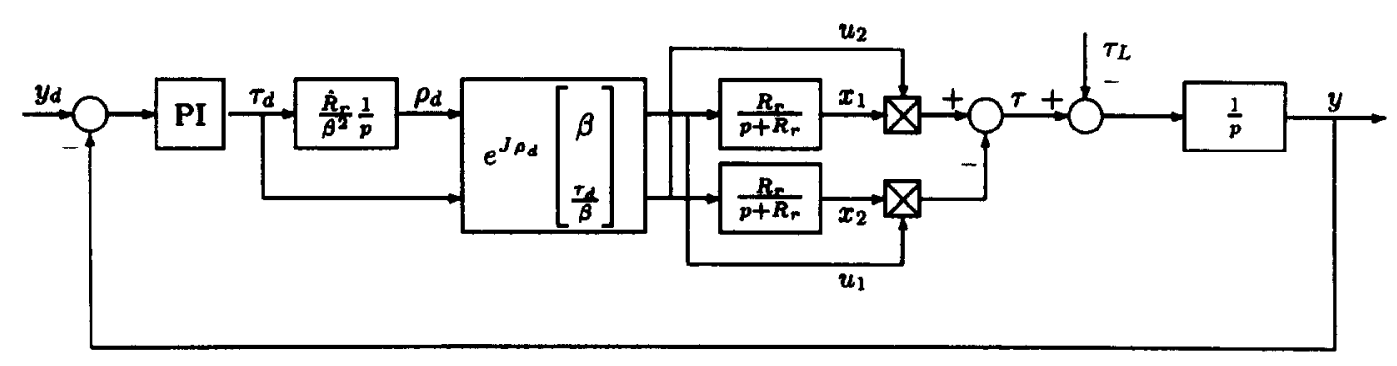

Fig. 1. Closed-loop velocity control of induction motor using indirect FOC.

$$
u=\mathrm{e}^{J \rho}\left[\begin{array}{l}
\beta \\
\frac{\tau_{\mathrm{d}}}{\beta}
\end{array}\right],
$$

with $\rho=\arctan \left(\frac{x_{2}}{x_{1}}\right)$ the rotor flux angle. In indirect FOC we simply replace $\rho$ by $\rho_{\mathrm{d}}$. Further, notice from (1)-(3) that, whenever $|x| \neq$ $0, \rho$ satisfies

$$
\dot{\rho}=\frac{R_{\mathrm{r}}}{|x|^{2}} \tau,
$$

which motivates the choice of $\rho_{\mathrm{d}}$ given in (5). That is, (5) follows replacing $|x|$ and $\tau$ by their desired values $\beta, \tau_{d}$, respectively, and replacing $R_{\mathrm{r}}$ by its estimate. In the electrical machines literature $\rho_{\mathrm{d}}$ is sometimes refered to as the slip angle. Therefore, expression (5) shows that the desired torque is proportional to the slip speed.

The remaining of the paper is organized as follows. In the next section we prove GAS for the case of known parameters. In Section 3 we represent the closed loop as the feedback interconnection of a linear time-invariant (LTI) system, a sector bounded nonlinearity and an exponentially decaying gain. This allows us, by invoking the small gain theorem (Desoer and Vidyasagar, 1975), to derive conditions for global boundedness. Necessary and sufficient conditions for the existence of a unique equilibrium are given in Section 4. Conditions for local and global asymptotic stability are derived using Lyapunov techniques in Sections 5 and 6, respectively. We wrap up the paper with some concluding remarks.

\section{GLOBAL ASYMPTOTIC STABILITY: KNOWN PARAMETER CASE}

Before proceeding with the study of the general case it is convenient, to set up the notation, to briefly recall here the GAS result of Ortega et al. (1995) for the case of known rotor resistance.

Proposition 1. Consider the system (1), (2), (3) in closed loop with (4), (5) and (6). If $\hat{R}_{\mathrm{r}}=R_{\mathrm{r}}$, then the system is GAS.
Proof. (Ortega et al., 1995) First, some simple calculations show that (4), (5) can be alternatively described with the (nonminimal) representation

$$
\begin{aligned}
\dot{x}_{\mathrm{d}} & =\frac{\hat{R}_{\mathrm{r}}}{\beta^{2}} \tau_{\mathrm{d}} J x_{\mathrm{d}}, x_{\mathrm{d}}(0)=\left[\begin{array}{l}
\beta \\
0
\end{array}\right], \\
u & =\left(I+\frac{\tau_{\mathrm{d}}}{\beta^{2}} J\right) x_{\mathrm{d}},
\end{aligned}
$$

with $I$ the $2 \times 2$ unity matrix. We interpret $x_{\mathrm{d}}$ as the desired rotor flux: $\left|x_{\mathrm{d}}\right|=\beta$ and $\tau_{\mathrm{d}}=u^{\mathrm{T}} J x_{\mathrm{d}}$, and

$$
x_{\mathrm{d}}=\beta\left[\begin{array}{c}
\cos \left(\rho_{\mathrm{d}}\right) \\
\sin \left(\rho_{\mathrm{d}}\right)
\end{array}\right] .
$$

Then, from (1), (7) and defining $\tilde{x}=x-x_{d}$ we see that

$$
\begin{aligned}
\dot{\tilde{x}} & =-R_{\mathrm{r}} \tilde{x}+\left(\frac{R_{\mathrm{r}}}{\hat{R}_{\mathrm{r}}}-1\right) \dot{x}_{\mathrm{d}}, \\
& =-R_{\mathrm{r}} \tilde{x}+\frac{1}{\beta}\left(R_{\mathrm{r}}-\hat{R}_{\mathrm{r}}\right) \tau_{\mathrm{d}} \mathrm{e}^{J \rho_{\mathrm{d}}}\left[\begin{array}{l}
0 \\
1
\end{array}\right] .
\end{aligned}
$$

From this equation, it is clear that $\tilde{x}$ converges to zero (exponentially) if $\hat{R}_{\mathrm{r}}=R_{\mathrm{r}}$.

Finally, defining $\tilde{y}=y-y_{\mathrm{d}}$ as the speed tracking error, and recalling that $y_{\mathrm{d}}$ is constant, we have from (2), (3) that

$$
\begin{aligned}
\dot{y}=\dot{y}= & u^{\mathrm{T}} J\left(\tilde{x}+x_{\mathrm{d}}\right)-\tau_{L} \\
= & x_{\mathrm{d}}^{\mathrm{T}} J \tilde{x}-\tau_{L}+\left(1+\frac{1}{\beta^{2}} x_{\mathrm{d}}^{\mathrm{T}} \tilde{x}\right) \tau_{\mathrm{d}} \\
= & -K_{P}\left(1+\frac{1}{\beta^{2}} x_{\mathrm{d}}^{\mathrm{T}} \tilde{x}\right) \tilde{y}-K_{I}\left(1+\frac{1}{\beta^{2}} x_{\mathrm{d}}^{\mathrm{T}} \tilde{x}\right) \frac{1}{p} \tilde{y} \\
& +x_{\mathrm{d}}^{\mathrm{T}} J \tilde{x}-\tau_{L},
\end{aligned}
$$

where we have used (8) and the skew-symmetry of $J$ to obtain the second identity, and we have replaced (6) to get the third one. Assuming again that $R_{\mathrm{r}}=$ $\hat{R}_{\mathrm{r}}$, and noting that $\left|x_{\mathrm{d}}(t)\right|=\beta$, we have that $\frac{1}{\beta^{2}} x_{\mathrm{d}}^{\mathrm{T}} \tilde{x}$ and $x_{\mathrm{d}}^{\mathrm{T}} J \tilde{x}$ are exponentially decaying terms. Asymptotic stability of $\tilde{y} \rightarrow 0$ follows invoking standard arguments of linear time-varying systems with exponentially convergent coefficients (Khalil, 1992).

In Section 4 we will give an alternative proof of GAS constructing a strict Lyapunov function. 


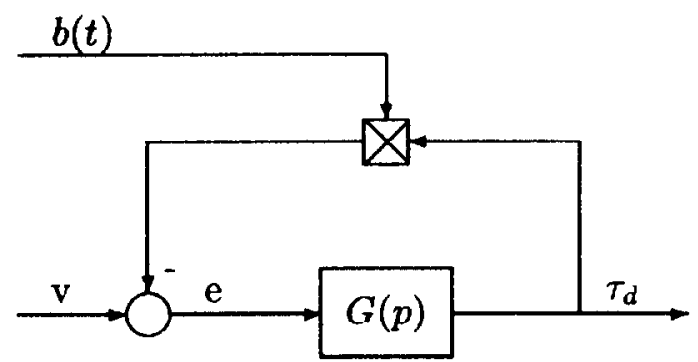

Fig. 2. Input-output description of closed-loop system.

\section{GLOBAL BOUNDEDNESS: INPUT-OUTPUT APPROACH}

In this section we use input-output techniques to derive sufficient conditions that ensure all signals of the closed loop remain uniformly bounded. The result is established showing that the closed-loop system can be viewed as a feedback interconnection of an LTI system, an exponentially decaying timevariant gain, and a time-variant sector bounded gain. The inputs of the closed-loop system are in $\mathcal{L}_{\infty}$, where $\mathcal{L}_{\infty}$ denotes the space of (essentially) bounded signals. Conditions for global boundedness are then obtained via a direct application of the $\mathcal{L}_{\infty}$ small gain theorem and of the fact that the feedback interconnection of a linear time-variant (LTV) system with finite $\mathcal{L}_{\infty}$-gain and a timevarying gain $b_{1}(t) \in \mathcal{L}_{1}$ has finite $\mathcal{L}_{\infty}$-gain (Desoer and Vidyasagar, 1975), where $\mathcal{L}_{1}$ is the space of signals $k(t)$ such that $\int_{0}^{\infty}\|k(t)\| \mathrm{d} t$ exists.

Proposition 2. The system (1)-(3) in closed loop with (4)-(6) may be written as (see Fig. 2)

$$
\begin{aligned}
\boldsymbol{T}_{\mathrm{d}} & =G(p) e, \\
e & =v-b(t) \tau_{\mathrm{d}} .
\end{aligned}
$$

where $G(p)$ is an LTI operator of the form

$$
G(p)=\frac{p K_{Y}+K_{I}}{p^{2}+\left(p K_{P}+K_{I}\right) \frac{R_{I}}{\hat{R}_{\mathrm{r}}}},
$$

with $v \in \mathcal{L}_{\infty}$ an external signal, and $b(t)=b_{\infty}(t)+$ $b_{1}(t)$ such that

$$
\begin{gathered}
\left|b_{\infty}(t)\right| \leq\left|\frac{\hat{R}_{\mathrm{r}}-R_{\mathrm{r}}}{\hat{R}_{\mathrm{r}}}\right|, \\
b_{1}(t) \in \mathcal{L}_{1} .
\end{gathered}
$$

The proof of the proposition, being a little technical, is deferred to the Appendix. Note that the feedback interconnection of an LTI system with gain $b_{\infty}(t)$ is an LTV system, for which sufficient conditions for finite $\mathcal{L}_{\infty}$-gain follow from the $\mathcal{L}_{\infty}$ Small Gain Theorem (Desoer and Vidyasagar, 1975). Also, a feedback interconnection of an LTV system with finite $\mathcal{L}_{\infty}$-gain

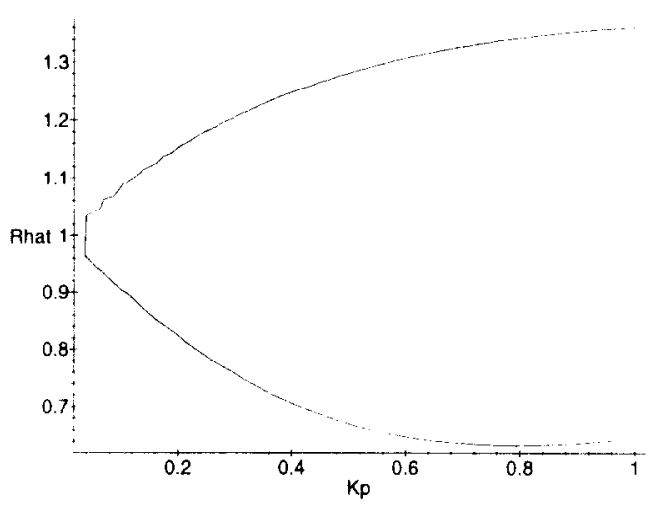

Fig. 3. Boundary of a region that guarantees boundedness for $K_{I}, R_{\mathrm{r}}=1$

and a feedback gain $b_{1}(t) \in \mathcal{L}_{1}$ has finite $\mathcal{L}_{\infty}$ gain (Desoer and Vidyasagar, 1975). This leads to the following result.

Corollary 1. All signals of the closed loop are bounded provided that $\hat{R}_{\mathrm{r}}$ is estimated close enough to $R_{\mathrm{r}}$.

\section{Discussion}

- A stronger condition for boundedness is obtained if the $\mathcal{L}_{1}$ gain of $G(p)$ is calculated. For example, if $K_{I}=0$, then the $\mathcal{L}_{\infty}$ gain of $G(p)$ is $\frac{R_{r}}{\tilde{R}_{r}}$. Then, the feedback interconnection of $G(p)$ and the $b(t)$ gain has finite $\mathcal{L}_{\infty}$ gain if $0<\hat{R}_{\mathrm{r}}<2 R_{\mathrm{r}}$. If $K_{I}=1, R_{\mathrm{r}}=1$ then a region for $\left(K_{P}, \hat{R}_{\mathrm{r}}\right)$ that guarantees boundedness is shown in Fig. 3.

- It is clear from the description of the system given in Proposition 2 that a wide variety of stability conditions can be derived, using, for instance, exponential weighting techniques and loop transformations. Results along these lines will be reported elsewhere.

\section{COORDINATE CHANGES AND UNIQUENESS OF EQUILIBRIUM}

To carry out the asymptotic stability analysis in the general case we find it convenient to work with a state space representation of the system. First, let us define the nonlinear coordinate transformation

$$
v=\left[\begin{array}{l}
v_{1} \\
v_{2} \\
v_{3} \\
v_{4}
\end{array}\right]=\left[\begin{array}{c}
x_{\mathrm{d}}^{\mathrm{T}} J x \\
x_{\mathrm{d}}^{\mathrm{T}} x \\
\mathrm{\tau}_{\mathrm{d}} \\
\tilde{y}
\end{array}\right] .
$$

This transformation is stability-preserving because it is invertible, and both the transformation and its inverse are differentiable. The transformation results in the following dynamic model: 


$$
\begin{aligned}
\dot{v}= & {\left[\begin{array}{cccc}
-R_{\mathrm{r}} & \hat{R}_{\mathrm{r}} \frac{v_{3}}{\beta^{2}} & -R_{\mathrm{r}} & 0 \\
-\hat{R}_{\mathrm{r}} \frac{v_{3}}{\beta^{2}} & -R_{\mathrm{r}} & 0 & 0 \\
-K_{P} & 0 & -K_{P} \frac{v_{2}}{\beta^{2}} & -K_{I} \\
1 & 0 & \frac{\nu_{2}}{\beta^{2}} & 0
\end{array}\right] v } \\
& +\left[\begin{array}{c}
0 \\
R_{\mathrm{r}} \beta^{2} \\
K_{P} \tau_{L} \\
-\tau_{L}
\end{array}\right] .
\end{aligned}
$$

Now, we shift the equilibrium to the origin. To this end, we define the new coordinates $w=v-\bar{v}$ where $\bar{v} \in \mathbb{R}^{4}$ is an equilibrium of (11). Below we will show that, for all practical purposes, the equilibrium is unique. The transformed dynamic model becomes

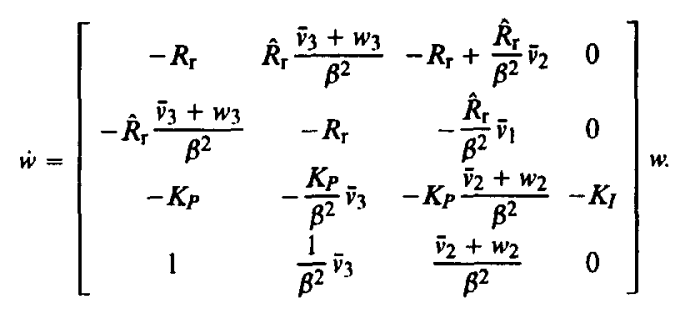

Now we will prove the following.

Proposition 3. The equilibria of (11) are independent of $K_{P}, K_{I}$. Further, the equilibrium is unique for all values of $\tau_{L}$ if and only if $0<\hat{R}_{\mathrm{r}} \leq 3 R_{\mathrm{r}}$.

Proof. The equilibria of (11) are all solutions $\bar{v} \in$ $\mathbb{R}^{4}$ to the equation

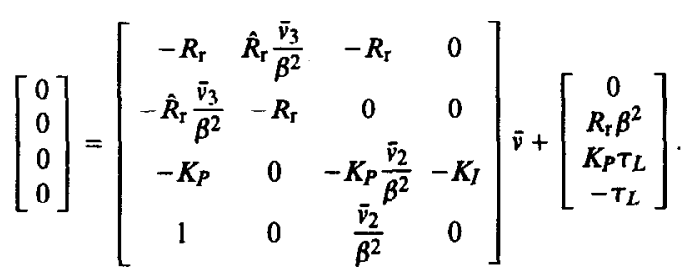

For any equilibrium point we must have $\bar{v}_{4}=0$ as can be seen from the third and fourth row of equation (11). This simplifies the equilibrium equations to

$$
\left[\begin{array}{l}
0 \\
0 \\
0
\end{array}\right]=\left[\begin{array}{ccc}
-R_{\mathrm{r}} & \hat{R}_{\mathrm{r}} \frac{\bar{v}_{3}}{\beta^{2}} & -R_{\mathrm{r}} \\
-\hat{R}_{\mathrm{r}} \frac{\bar{v}_{3}}{\beta^{2}} & -R_{\mathrm{r}} & 0 \\
-K_{P} & 0 & -K_{P} \frac{\bar{v}_{2}}{\beta^{2}}
\end{array}\right]\left[\begin{array}{c}
\bar{v}_{1} \\
\bar{v}_{2} \\
\bar{v}_{3}
\end{array}\right]+\left[\begin{array}{c}
0 \\
R_{\mathrm{r}} \beta^{2} \\
K_{P} \tau_{L}
\end{array}\right] .
$$

Simple algebraic manipulations lead to the following third-order polynomial in $\bar{v}_{3}$ :

$$
R_{\mathrm{r}} \hat{R}_{\mathrm{r}} \bar{v}_{3}^{3}-\hat{R}_{\mathrm{r}}^{2} \tau_{L} \bar{\nu}_{3}^{2}+R_{\mathrm{r}} \hat{R}_{\mathrm{r}} \beta^{4} \bar{v}_{3}-R_{\mathrm{r}}^{2} \beta^{4} \tau_{L}=0 .
$$

If the equilibrium value of $\bar{v}_{3}$ is known, then $\bar{v}_{1}$ and $\bar{v}_{2}$ can be calculated using

$$
\bar{v}_{1}=-\frac{\left(R_{\mathrm{r}}-\hat{R}_{\mathrm{r}}\right) \beta^{4} R_{\mathrm{r}} \bar{v}_{3}}{R_{\mathrm{r}}^{2} \beta^{4}+\hat{R}_{\mathrm{r}}^{2} \bar{v}_{3}^{2}},
$$

$$
R_{\mathrm{r}} \bar{v}_{2}=\frac{\left(R_{\mathrm{r}} \beta^{4}+\hat{R}_{\mathrm{r}} \bar{\nu}_{3}^{2}\right) R_{\mathrm{r}} \beta^{2}}{R_{\mathrm{r}}^{2} \beta^{4}+\hat{R}_{\mathrm{r}}^{2} \bar{\nu}_{3}^{2}}
$$

Henceforth, we will concentrate on the real solutions of (13). In particular we will investigate the conditions under which the function $T_{L}=T_{L}\left(\bar{v}_{3}\right)$ is bijective, i.e. $\bar{v}_{3}$ is also a function of $\tau_{L}$.

The expression for $\tau_{L}$ as a function of $\bar{v}_{3}$ is

$$
\tau_{L}=\frac{R_{\mathrm{r}} \hat{R}_{\mathrm{r}} \bar{v}_{3}^{3}+R_{\mathrm{r}} \hat{R}_{\mathrm{r}} \beta^{4} \bar{v}_{3}}{R_{\mathrm{r}}^{2} \beta^{4}+\hat{R}_{\mathrm{r}}^{2} \bar{\nu}_{3}^{2}} .
$$

Clearly, $\tau_{L}\left(\bar{v}_{3}\right)$ is continuous and subjective. Then, it is a bijection if it is strictly monotonic. The derivative of $T_{L}\left(\bar{v}_{3}\right)$ is

$$
\begin{aligned}
& \frac{\mathrm{d} \tau_{L}}{\mathrm{~d} \bar{v}_{3}}= \\
& \frac{R_{\mathrm{r}} \hat{R}_{\mathrm{r}}^{3} \bar{v}_{3}^{4}+\left(3 R_{\mathrm{r}}^{3} \hat{R}_{\mathrm{r}} \beta^{4}-R_{\mathrm{r}} \hat{R}_{\mathrm{r}}^{3} \beta^{4}\right) \bar{v}_{3}^{2}+R_{\mathrm{r}}^{3} \hat{R}_{\mathrm{r}} \beta^{8}}{\left(R_{\mathrm{r}}^{2} \beta^{4}+\hat{R}_{\mathrm{r}}^{2} \bar{v}_{3}^{2}\right)^{2}} .
\end{aligned}
$$

The denominator in this equation is always positive. Therefore, if the numerator is of constant sign, $\tau_{L}\left(\bar{v}_{3}\right)$ is bijective. The numerator of $(16)$ is a polynomial in $\vec{v}_{3}^{2}$. This polynomial is of constant sign if its discriminant is less than or equal to zero, that is, if

$$
9 R_{\mathrm{r}}^{4}-10 R_{\mathrm{r}}^{2} \hat{R}_{\mathrm{r}}^{2}+\hat{R}_{\mathrm{r}}^{4} \leq 0 .
$$

The discriminant is a polynomial in $\hat{R}_{\mathrm{r}}^{2}$ which is less than or equal to zero for $\hat{R}_{\mathrm{r}}^{2} \in\left[R_{\mathrm{r}}^{2}, 9 R_{\mathrm{r}}^{2}\right]$.

Also, if $\hat{R}_{\mathrm{r}}<\sqrt{3} R_{\mathrm{r}}$ then all terms in the numerator in (16) are strictly positive. Then, $\tau_{L}$ is a monotonic function of $\bar{v}_{3}$. If, on the other hand, $\hat{R}_{\mathrm{r}}>3 R_{\mathrm{r}}$ then values for $\bar{v}_{3}$ can be found where $\frac{d \tau_{L}}{d \bar{v}_{3}}<0$, so that $\tau_{L}$ as a function of $\bar{v}_{3}$ is not monotonic anymore and therefore not bijective.

From (14)-(15), the following bounds on $\bar{v}_{1}, \bar{v}_{2}$ can be calculated, which will be used in Section 6:

$$
\begin{gathered}
\left|\bar{v}_{1}\right| \leq \frac{1}{2}\left|\frac{R_{\mathrm{r}}-\hat{R}_{\mathrm{r}}}{\hat{R}_{\mathrm{r}}}\right| \beta^{2}, \\
R_{\mathrm{r}}>\hat{R}_{\mathrm{r}} \Rightarrow \beta^{2} \leq \bar{v}_{2} \leq \frac{R_{\mathrm{r}}}{\hat{R}_{\mathrm{r}}} \beta^{2}, \\
R_{\mathrm{r}} \leq \hat{R}_{\mathrm{r}} \Rightarrow \frac{R_{\mathrm{r}}}{\hat{R}_{\mathrm{r}}} \beta^{2} \leq \bar{v}_{2} \leq \beta^{2} .
\end{gathered}
$$

As an example of the existence of multiple equilibria for certain ranges of $\tau_{L}$, the roots of (13) will now be determined by application of the root locus technique to the more suitable form of (13).

$$
1-\tau_{L} \frac{\hat{R}_{\mathrm{r}}}{R_{\mathrm{r}}} \frac{\bar{v}_{3}^{2}+\frac{R_{\mathrm{r}}^{2}}{\bar{R}_{2}^{2}} \beta^{4}}{\left.\bar{v}_{3}^{2}+\beta^{4}\right)}=0
$$




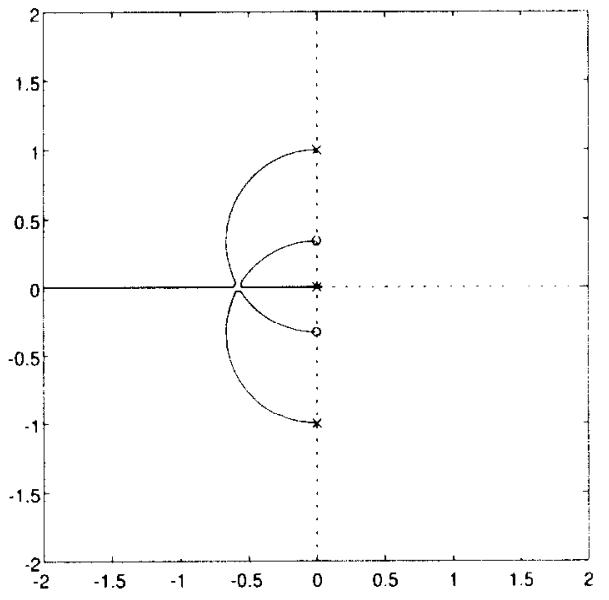

Fig. 4. Root locus of the system equilibria for $\hat{R}_{\mathrm{r}}=3 R_{\mathrm{r}}$

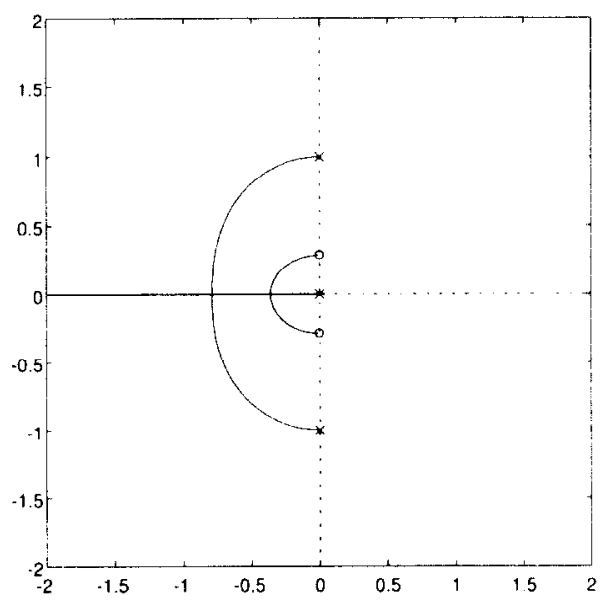

Fig. 5. Root locus of the system equilibria for $\hat{R}_{\mathrm{r}}>3 R_{\mathrm{r}}$.

The uniqueness of equilibrium for $\hat{R}_{\mathrm{r}}=3 R_{\mathrm{r}}$ is evident from Fig. 4, since there are three coinciding real roots for one value of $\tau_{L}$, while for any other value of $\tau_{L}$ there is only one real root. The nonuniqueness of the equilibria for $\hat{R}_{\mathrm{r}}>3 R_{\mathrm{r}}$ causes the locus of Fig. 5 to have three distinct real roots for a certain range of $\tau_{L}$. If $\hat{R}_{\mathrm{r}}<3 R_{\mathrm{r}}$ then the two poles go directly to the zero without crossing the real axis.

Before closing this Section it is interesting to 'pull out' the nonlinear terms of (12) as

$$
\begin{aligned}
\dot{w}= & {\left[\begin{array}{cccc}
-R_{\mathrm{r}} & \hat{R}_{\mathrm{r}} \frac{\bar{v}_{3}}{\beta^{2}} & -R_{\mathrm{r}}+\frac{\hat{R}_{\mathrm{r}}}{\beta^{2}} \bar{v}_{2} & 0 \\
-\hat{R}_{\mathrm{r}} \frac{\bar{v}_{3}}{\beta^{2}} & -R_{\mathrm{r}} & -\frac{\hat{R}_{\mathrm{r}}}{\beta^{2}} \bar{v}_{1} & 0 \\
-K_{P} & -\frac{K_{P}}{\beta^{2}} \bar{v}_{3} & -K_{P} \frac{\bar{v}_{2}}{\beta^{2}} & -K_{I} \\
1 & \frac{1}{\beta^{2}} \bar{v}_{3} & \frac{\bar{v}_{2}}{\beta^{2}} & 0
\end{array}\right] w } \\
& +\left[\begin{array}{c}
\hat{R}_{\mathrm{r}} w_{2} \\
-\hat{R}_{\mathrm{r}} w_{1} \\
-K_{P} w_{2} \\
w_{2}
\end{array}\right] \frac{w_{3}}{\beta^{2}} .
\end{aligned}
$$

Noting the presence in the right-hand term of the scaling factor $\frac{w_{3}}{\beta^{2}}$ and referring to Fig. 1 we see from (19) that, roughly speaking, the closed-loop system behaves 'almost linearly' if the PI speed loop is not too tight. That is, if $\frac{w_{3}}{\beta^{2}}$ is 'small' and/or slowly time varying.

\section{LOCAL ASYMPTOTIC STABILITY}

In this section we will study, via the first Lyapunov method, the local asymptotic stability of (12). Towards this end, we see that the systems firstorder approximation is simply the first right-hand term of (19), whose characteristic polynomial is

$$
\left|\begin{array}{cccc}
s+R_{\mathrm{r}} & -\hat{R}_{\mathrm{r}} \frac{\bar{\nu}_{3}}{\beta^{2}} & R_{\mathrm{r}}-\frac{\hat{R}_{\mathrm{r}}}{\beta^{2}} \bar{\nu}_{2} & 0 \\
\hat{R}_{\mathrm{r}} \frac{\bar{v}_{3}}{\beta^{2}} & s+R_{\mathrm{r}} & \frac{\hat{R}_{\mathrm{r}}}{\beta^{2}} \bar{v}_{1} & 0 \\
K_{P} & \frac{K_{P}}{\beta^{2}} \bar{\nu}_{3} & s+K_{P} \frac{\bar{\nu}_{2}}{\beta^{2}} & K_{I} \\
-1 & -\frac{1}{\beta^{2}} \bar{v}_{3} & -\frac{\bar{v}_{2}}{\beta^{2}} & s
\end{array}\right|=0 .
$$

Given the complexity of the expression above (recall that $\bar{v}$ is itself a nonlinear function of the motor parameters) we are unable at this point to make a general statement concerning the stability of the roots of this polynomial. Consequently, we will only consider below some special cases. In particular, we will show that, even with zero load torque, the equilibrium may become unstable.

\section{Known parameter case.}

As shown before when $R_{\mathrm{r}}=\hat{R}_{\mathrm{r}}$ the equilibrium is GAS. However, to provide some tuning rules it is interesting to look at the behaviour of the roots of the linearized system, for instance, as a function of the load torque. To this end, we write the characteristic polynomial in a $\tau_{L}$-root locus form as

$$
\left(1+\frac{\tau_{L}^{2} R_{\mathrm{r}}^{2}}{\beta^{4}} \frac{1}{\left(s+R_{\mathrm{r}}\right)^{2}}\right)\left(s^{2}+K_{P} s+K_{I}\right)=0 .
$$

The closed loop then has two poles at fixed positions determined by $K_{P}, K_{I}$ and, as $\tau_{L}$ increases, 
the double pole at $s=-R_{\mathrm{r}}$ moves along straight asymptotes in the left half plane.

Proposition 4. Local stability in zero load torque case. Assume $\tau_{L}=0$. Then, the system is locally asymptotically stable if $K_{P}^{2}>K_{I}$. On the other hand, the equilibrium will be unstable if $\hat{R}_{\mathrm{r}}>$ $R_{\mathrm{r}}+K_{P}$ and a large integral gain is used.

Proof. Note that $\tau_{L}=0$ implies a unique equilibrium with $\bar{w}=0, \bar{v}_{1}=0, \bar{v}_{2}=\beta^{2} \bar{v}_{3}=0$ and $\bar{v}_{4}=0$. Then, the characteristic equation reduces to

$$
\begin{aligned}
& \left(s+R_{\mathrm{r}}\right)^{2}\left(s\left(s+K_{P}\right)+K_{I}\right)+K_{P}\left(s+R_{\mathrm{r}}\right)\left(\hat{R}_{\mathrm{r}}-R_{\mathrm{r}}\right) s \\
& \quad+\left(s+R_{\mathrm{r}}\right)\left(\hat{R}_{\mathrm{r}}-R_{\mathrm{r}}\right) K_{I}=0 .
\end{aligned}
$$

The proof is completed noting that this equation has one root at $s=-R_{\mathrm{r}}$, while from Routh-Hurwitz we know that the other roots are on the open the left-hand plane if and only if

$$
\hat{R}_{\mathrm{r}} R_{\mathrm{r}} K_{P}+\hat{R}_{\mathrm{r}} K_{P}^{2}>\left(\hat{R}_{\mathrm{r}}-R_{\mathrm{r}}-K_{P}\right) K_{I} .
$$

Therefore, the equilibrium is unstable if $\hat{R}_{\mathrm{r}}-R_{\mathrm{r}}-$ $K_{P}>0$ and $K_{I}$ is sufficiently large. Also, this condition implies that the system is locally asymptotically stable if $K_{P}^{2}>K_{I}$.

The proposition above shows that one can choose PI-controller gains that guarantee local asymptotic stability for all values of the rotor resistance. Also, it shows that the system can be destabilized, in the sense of having unstable equilibria; if the rotor resistance is overestimated, the proportional gain is small, and a large integral gain is used. For example, if $y_{\mathrm{d}}=0, \tau_{L}=0, R=1, \hat{R}=4, K_{P}=1$ and $K_{I}=6$ then the system is unstable (Fig. 6).

\section{GLOBAL ASYMPTOTIC STABILITY}

In this section we will investigate GAS of the equilibrium using Lyapunov's second method. Namely, we will construct a Lyapunov function of the form

$$
V(w)=\frac{1}{2} w^{\mathrm{T}} P w,
$$

where $P$ is a positive definite constant matrix. To select $P$ we first find positive semi-definite matrices $P_{i}$ that lead to expressions without cubic terms in the derivative of $V$. Second, linear combinations of these positive semi-definite matrices are constructed that lead to a negative definite $\dot{V}(w)$ if $R_{\mathrm{r}}=\hat{R}_{\mathrm{r}}$. Then, the positive definiteness of $P$ is checked. Finally, we prove that the system is globally asymptotically stable if $\hat{R}_{\mathrm{r}}$ is close enough to $R_{\mathrm{r}}$.
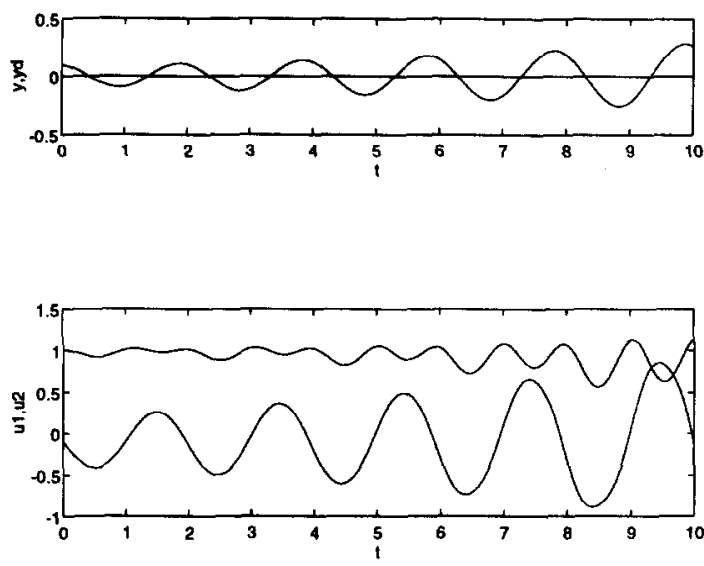

Fig. 6. Instability caused by overestimation of $R$ and small stability margin.

\subsection{Positive semi-definite matrices to avoid cubic terms}

To construct our Lyapunov-function candidate we consider for $P$ linear combinations of the following positive semi-definite matrices $P_{i}, i=1, \ldots, 4$.

$$
\begin{gathered}
P_{1}=\left[\begin{array}{llll}
1 & 0 & 0 & 0 \\
0 & 1 & 0 & 0 \\
0 & 0 & 0 & 0 \\
0 & 0 & 0 & 0
\end{array}\right], P_{2}=\left[\begin{array}{cccc}
\frac{1}{\hat{R}_{\mathrm{r}}} & 0 & 0 & -1 \\
0 & 0 & 0 & 0 \\
0 & 0 & 0 & 0 \\
-1 & 0 & 0 & \hat{R}_{\mathrm{r}}
\end{array}\right], \\
P_{3}=\left[\begin{array}{llll}
0 & 0 & 0 & 0 \\
0 & 0 & 0 & 0 \\
0 & 0 & 1 & K_{P} \\
0 & 0 & K_{P} & K_{P}^{2}
\end{array}\right], P_{4}=\left[\begin{array}{cccc}
K_{P}^{2} & 0 & K_{P} \hat{R}_{\mathrm{r}} & 0 \\
0 & 0 & 0 & 0 \\
K_{P} \hat{R}_{\mathrm{r}} & 0 & \hat{R}_{\mathrm{r}}^{2} & 0 \\
0 & 0 & 0 & 0
\end{array}\right] .
\end{gathered}
$$

The corresponding functions $V_{i}(w)=\frac{1}{2} w^{\mathrm{T}} P_{i} w, i=$ $1, \ldots, 4$ have derivatives

$$
\begin{aligned}
\dot{V}_{1}(w)= & w^{\mathrm{T}} P_{1} \dot{w} \\
= & -R_{\mathrm{r}} w_{1}^{2}-\frac{R_{\mathrm{r}} \beta^{2}-\hat{R}_{\mathrm{r}} \bar{v}_{2}}{\beta^{2}} w_{1} w_{3} \\
& -R_{\mathrm{r}} w_{2}^{2}-\frac{\hat{R}_{\mathrm{r}} \bar{v}_{1}}{\beta^{2}} w_{2} w_{3}, \\
\dot{V}_{2}(w)= & w^{\mathrm{T}} P_{2} \dot{w} \\
= & -\frac{R_{\mathrm{r}}+\hat{R}_{\mathrm{r}}}{\hat{R}_{\mathrm{r}}} w_{1}^{2}-\frac{R_{\mathrm{r}}}{\hat{R}_{\mathrm{r}}} w_{1} w_{3} \\
& +\left(\hat{R}_{\mathrm{r}}+R_{\mathrm{r}}\right) w_{1} w_{4}+R_{\mathrm{r}} w_{3} w_{4}, \\
\dot{V}_{3}(w)= & w^{\mathrm{T}} P_{3} \dot{w}=-K_{I} K_{P} w_{4}^{2}-K_{I} w_{3} w_{4}, \\
\dot{V}_{4}(w)= & w^{\mathrm{T}} P_{4} \dot{w} \\
= & -K_{P}^{2}\left(R_{\mathrm{r}}+\hat{R}_{\mathrm{r}}\right) w_{1}^{2} \\
& -\left(K_{P} \hat{R}_{\mathrm{r}}\left(R_{\mathrm{r}}+\hat{R}_{\mathrm{r}}\right)+K_{P}^{2} R_{\mathrm{r}}\right) w_{1} w_{3} \\
& -K_{P} K_{I} \hat{R}_{\mathrm{r}} w_{1} w_{4}-K_{P} R_{\mathrm{r}} \hat{R}_{\mathrm{r}} w_{3}^{2} \\
& -K_{I} \hat{R}_{\mathrm{r}}^{2} w_{3} w_{4} .
\end{aligned}
$$


Since these derivatives have only quadratic terms, the derivative of $V(w)=w^{\mathrm{T}} P w$ will also have only quadratic terms if $P$ is a linear combination of $P_{i}, i=1, \ldots, 4$. As a consequence, the global negative definiteness of $\dot{V}(w)$ can be easily checked.

\subsection{Construction of a Lyapunov function}

Proposition 5. There is a positive definite constant matrix $P$ such that $V(w)=\frac{1}{2} w^{\mathrm{T}} \boldsymbol{P} w$ is a Lyapunov function for the system (12) with $\bar{v}_{1}, \bar{v}_{2}, \bar{v}_{3}$ the solutions of (13)-(15), if $\hat{R}_{\mathrm{r}}=R_{\mathrm{r}}$, or if $\hat{R}_{\mathrm{r}}$ is close enough to $R_{\mathrm{r}}$.

Proof. We begin the proof by constructing a Lyapunov function for the case $\hat{R}_{\mathrm{r}}=R_{\mathrm{r}}$. Then, we will show that this function is also a Lyapunov function if $\hat{R}_{\mathrm{r}}$ is close enough to $R_{\mathrm{r}}$.

If $\hat{R}_{\mathrm{r}}=R_{\mathrm{r}}$, the cross-terms in $\dot{V}_{1}(w)$ dissappear because $\bar{v}_{1}=0$ and $\bar{v}_{2}=\beta^{2}$ in equilibrium. Consider the matrix

$$
P_{a}=P_{3}+P_{4}
$$

This choice of $P_{a}$ results in the candidate Lyapunov function $V_{a}(w)=\frac{1}{2} w^{\mathrm{T}} P_{a} w$ with derivative

$$
\begin{aligned}
\dot{V}_{a}(w)= & -2 K_{P}^{2} R_{\mathrm{r}} w_{1}^{2}-R_{\mathrm{r}}^{2} K_{P} w_{3}^{2}-K_{I} K_{P} w_{4}^{2} \\
& -\left(2 K_{P} R_{\mathrm{r}}^{2}+K_{P}^{2} R_{\mathrm{r}}\right) w_{1} w_{3}-K_{P} K_{I} R_{\mathrm{r}} w_{1} w_{4} \\
& -\left(K_{I}+R_{\mathrm{r}}^{2} K_{I}\right) w_{3} w_{4} .
\end{aligned}
$$

The cross-term in $w_{3} w_{4}$ can be cancelled by adding a term in $P_{2}$ to $P_{a}$

$$
P_{h}=P_{3}+P_{4}+\frac{K_{I}+R_{\mathrm{r}}^{2} K_{I}}{R_{\mathrm{r}}} P_{2},
$$

which results in the candidate Lyapunov function $V_{b}(w)$ with derivative

$$
\begin{aligned}
\dot{V}_{h}(w)= & -2\left(K_{P}^{2} R_{\mathrm{r}}+\frac{K_{I}+R_{\mathrm{r}}^{2} K_{I}}{R_{\mathrm{r}}}\right) w_{\mathrm{l}}^{2} \\
& -R_{\mathrm{r}}^{2} K_{P} w_{3}^{2}-K_{I} K_{P} w_{4}^{2} \\
& -\left(2 K_{P} R_{\mathrm{r}}^{2}+K_{P}^{2} R_{\mathrm{r}}+\frac{K_{I}+R_{\mathrm{r}}^{2} K_{I}}{R_{\mathrm{r}}}\right) w_{1} w_{3} \\
& +\left(2\left(K_{I}+R_{\mathrm{r}}^{2} K_{I}\right)-K_{P} K_{I} R_{\mathrm{r}}\right) w_{1} w_{4} \\
= & -a_{1} w_{1}^{2}-a_{3} w_{3}^{2}-a_{4} w_{4}^{2} \\
& -2 b_{13} w_{1} w_{3}+2 b_{14} w_{1} w_{4} .
\end{aligned}
$$

This derivative can always be rendered negative definite by adding a component $\left(z_{3}+z_{4}\right) P_{1}$ to the matrix $P_{h}$

$$
P=P_{3}+P_{4}+\frac{K_{I}+R_{\mathrm{r}}^{2} K_{1}}{R_{\mathrm{r}}} P_{2}+\left(z_{3}+z_{4}\right) P_{1},
$$

where the coefficients $z_{3}, z_{4}$ are chosen to compensate for the cross-terms as follows:

$$
\begin{aligned}
& z_{3}=\frac{1}{R_{\mathrm{r}}} \frac{b_{13}^{2}}{a_{3}}, \\
& z_{4}=\frac{1}{R_{\mathrm{r}}} \frac{b_{14}^{2}}{a_{4}},
\end{aligned}
$$

so that the derivative of the Lyapunov function $V(w)=\frac{1}{2} w^{\mathrm{T}} P w$ becomes

$$
\begin{aligned}
\dot{V}(w)= & -a_{1} w_{1}^{2} \\
& -a_{3} w_{3}^{2}-a_{4} w_{4}^{2}-2 b_{13} w_{1} w_{3}+2 b_{14} w_{1} w_{4} \\
& -\frac{b_{13}^{2}}{a_{3}} w_{1}^{2}-\frac{b_{14}^{2}}{a_{4}} w_{1}^{2} \\
& -\left(\frac{b_{13}^{2}}{a_{3}}+\frac{b_{14}^{2}}{a_{4}}\right) w_{2}^{2} .
\end{aligned}
$$

The function $V(w)$ is positive definite and its derivative is negative definite. Therefore, $V(w)$ is a strict Lyapunov function for $R_{\mathrm{r}}=\hat{R}_{\mathrm{r}}$

To prove that $V(w)$ is a Lyapunov function also if $R_{\mathrm{r}} \neq \hat{R}_{\mathrm{r}}$ and $\hat{R}_{\mathrm{r}}$ close enough to $R_{\mathrm{r}}$, we write for the derivative

$$
\dot{V}(w)=-w^{\mathrm{T}} Q w
$$

and prove that $Q$ is positive definite if $\left|R_{\mathrm{r}}-\hat{R}_{\mathrm{r}}\right|$ is small enough.

A necessary and sufficient condition for a symmetric matrix $Q$ to be positive definite is that all its leading principal minors are positive. The leading principle minors of $Q$ are continuous functions of the elements of $Q$. The elements of $Q$ depend continuously on the coefficients of the polynomials $\dot{V}_{i}(w), \quad i=1, \ldots, 4$. The coefficients of $\dot{V}_{i}(w), \quad i=$ $2, \ldots, 4$ are continuous functions of $\hat{R}_{\mathrm{r}}$. From (17)(18), the following bounds on the coefficients of the cross-terms in $\dot{V}_{1}(w)$ are obtained:

$$
\begin{aligned}
\left|\frac{\hat{R}_{\mathrm{r}} \bar{v}_{1}}{\beta^{2}}\right| & \leq \frac{1}{2}\left|R_{\mathrm{r}}-\hat{R}_{\mathrm{r}}\right| . \\
\left|\frac{R_{\mathrm{r}} \beta^{2}-\hat{R}_{\mathrm{r}} \bar{v}_{2}}{\beta^{2}}\right| & \leq\left|R_{\mathrm{r}}-\hat{R}_{\mathrm{r}}\right| .
\end{aligned}
$$

Therefore, these coefficients are continuous functions of $\hat{R}_{\mathrm{r}}$ around $\hat{R}_{\mathrm{r}}=R_{\mathrm{r}}$. Therefore, the leading principal minors of $Q$ are continuous functions of $\hat{R}_{\mathrm{r}}$ around $\hat{R}_{\mathrm{r}}=R_{\mathrm{r}}$, so that $Q$ is positive definite if $\hat{R}_{\mathrm{r}}$ is close enough to $R_{\mathrm{r}}$.

\section{Discussion}

- Necessary and sufficient conditions for a function $V(w)=w^{\mathrm{T}} P w$ to be a Lyapunov function for the general system (12), with $P=\Sigma c_{i} P_{i}$ and $c_{i}>0$, have been derived but they are not given here since the conditions are very complicated.

- If the function $V(w)$ constructed in the proof of Proposition 5 is not a Lyapunov function 
for certain $\hat{R}_{\mathrm{r}} \neq R_{\mathrm{r}}$, other candidate Lyapunov functions can be constructed similarly to investigate stability.

- The robustness of practical systems with respect to rotor resistance estimation can be investigated with a numerical approach. For example, consider the parameter values $K_{P}=1$, $K_{I}=0.5, R_{\mathrm{r}}=1, \hat{R}_{\mathrm{r}}=1.6$ and arbitrary $\tau_{L}$ and $\beta$. Then, a Lyapunov function is

$$
V(w)=w^{\mathrm{T}}\left(4 P_{1}+K_{I} \frac{1+R_{\mathrm{r}}^{2}}{R_{\mathrm{r}}} P_{2}+P_{3}+P_{4}\right) w .
$$

This function is positive definite, and its derivative is negative definite because

$$
\dot{V}(w)=-w^{\mathrm{T}} Q\left(\bar{v}_{1}, \bar{v}_{2}\right) w,
$$

with $Q\left(\bar{v}_{1}, \bar{v}_{2}\right)$ a matrix whose off-diagonal elements depend on $\bar{v}_{1}, \bar{v}_{2}$ such that the leading principal minors of $Q$ are positive for all possible values of $\bar{v}_{1}, \bar{v}_{2}$.

\section{CONCLUSIONS}

We have shown that the widely used indirect fieldoriented control is globally asymptotically stable if the estimated rotor resistance estimate is estimated close enough to the real value. Unique equilibria are guaranteed if the estimated rotor resistance is within a $200 \%$ error range. Also, all signals in the system remain uniformly bounded if the estimated rotor resistance is close enough to the real rotor resistance. Particularly, with a sufficiently small integral gain, all signals are uniformly bounded if the rotor resistance is estimated within a $100 \%$ error range. The system becomes locally unstable if the rotor resistance is overestimated and a large integral gain is used. Local asymptotic stability is guaranteed if the square of the proportional gain is larger than the integral gain.

Acknowledgements - This work was supported in part by the Commission of European Communitics under contract ERB CHRX CT 93-0380. The critical remarks by Per J. Nicklasson were greatly appreciated by the authors. Part of this work was carried out while Romeo Ortega was visiting the Australian National University. Another part of this work was carried out while Paul de Wit was visiting Université de Technologie de Compiègne. The hospitality of these institutions is gratefully acknowledged.

\section{REFERENCES}

Bodson, M., J. Chiasson and R. Novotnak (1994). Highperformance induction motor control via input-output linearization. IEEE Control Systems Magazine.

Bose, B. K. (1986). Power Electronics and DC Drives. Prentice Hall, Englewood Cliffs, NJ.

Chiasson, J. (1993). Dynamic feedback linearization of the induction motor. IEEE Trans. Automat. Control, 338(10), 1588-1594.
Desoer. C. A. and M. Vidyasagar (1975). Feedback Systems: Input-Output Properties. Academic Press, New York.

Hughes, A., J. Corda and D. A. Andrade (1993). An inside look at cage motors with vector control. In Proc. 6th Int Conf. on Elec. Machines and Drives. IEE.

Kanellakopoulos, I., P. Krein and F. Disilvestro (1992). Nonlinear flux-observer-based control of induction motors. In Proc. ACC'92. Chicago, IL, U.S.A.

Khalil, H. (1992). Nonlinear Systems. Macmillan, NJ.

Kim, D., I. Ha and M. Ko (1990). Control of induction motors via feedback linearization with input-output decoupling. Int. J. Control, 51(4), 863-883.

Leonhard, W. (1985). Control of Electrical Drives. Springer, Berlin.

Marino, R., S. Peresada and P. Valigi (1993). Adaptive partial feedback linearization of induction motors. IEEE Trans. Automat. Control, 38(2), 208-221.

Ortega, R. and G. Espinosa (1993). Torque regulation of induction motors. Automatica, 29(3), 621-633.

Ortega, R., D. Taoutaou, R. Rabinovici and J. P. Vilain (1995). On field oriented and passivity-based control of induction motors: downward compatibility. In Proc. IFAC NOLCOS Conf., Tahoe city, CA.

Ortega, R., P. J. Nicklasson and G. Espinosa (1996). On speed control of induction motors. Automatica, 32(3) 455-460.

Taylor, D. (1994). Nonlinear control of electric machines: an overview. IEEE Control Systems Magazine, 14(6), 41-51.

\section{APPENDIX A. PROOF OF PROPOSITION 2}

Starting from (6) we have

$$
\begin{aligned}
\dot{\mathrm{T}}_{\mathrm{d}} & =-\left(K+P+\frac{K_{I}}{p}\right) \dot{y} \\
& =-\left(K+P+\frac{K_{I}}{p}\right)\left(u^{\mathrm{T}} J x_{\mathrm{d}}+u^{\mathrm{T}} J \tilde{x}-\tau_{L}\right) \\
& =-\left(K+P+\frac{K_{I}}{p}\right)\left(\tau_{\mathrm{d}}-\tau_{L}-\left[-\frac{\tau_{\mathrm{d}}}{\beta} \beta\right] \mathrm{e}^{\left.-J \rho_{\mathrm{d}} \tilde{x}\right) .}\right.
\end{aligned}
$$

The rest of the derivation is expressing $\mathrm{e}^{-J_{\rho_{\mathrm{d}}} \tilde{x}}$ as a bounded signal, which ensures a bounded nonlinear feedback since it is multiplied by $\tau_{d}$.

\section{A. 1. Boundedness of $\mathrm{e}^{-J \rho_{\mathrm{d}}} \tilde{x}$}

The definition of $\tilde{x}$ is $\tilde{x}=x-x_{\mathrm{d}}$ and $\tilde{R}_{\mathrm{r}}=\hat{R}_{\mathrm{r}}-R_{\mathrm{r}}$ so that the derivative becomes

$$
\begin{aligned}
\dot{\tilde{x}} & =\dot{x}-\dot{x}_{\mathrm{d}} \\
& =-R_{\mathrm{r}}\left(x-x_{\mathrm{d}}\right)-\tilde{R}_{\mathrm{r}} u+\tilde{R}_{\mathrm{r}} x_{\mathrm{d}} \\
& =-R_{\mathrm{r}} \tilde{x}+\tilde{R}_{\mathrm{r}}\left(x_{\mathrm{d}}-u\right) .
\end{aligned}
$$

The factor $x_{d}-u$ can be replaced

$$
x_{\mathrm{d}}-u=-\frac{1}{\hat{R}_{\mathrm{r}}} \dot{x}_{\mathrm{d}}=-\frac{1}{\beta^{2}} \tau_{\mathrm{d}} J x_{\mathrm{d}},
$$

so that

$$
\dot{\tilde{x}}=-R_{\mathrm{r}} \tilde{x}-\tilde{R}_{\mathrm{r}} \frac{1}{\beta^{2}} \tau_{\mathrm{d}} J x_{\mathrm{d}},
$$

$J x_{\mathrm{d}}$ is bounded

$$
J x_{\mathrm{d}}=J \beta \mathrm{e}^{J \rho_{\mathrm{d}}}\left[\begin{array}{l}
1 \\
0
\end{array}\right]=\beta \mathrm{e}^{J \rho_{\mathrm{d}}}\left[\begin{array}{l}
0 \\
1
\end{array}\right],
$$

so that the derivative of $\tilde{x}$ becomes 


$$
\dot{\tilde{x}}=-R_{\mathrm{r}} \tilde{x}-\frac{\tilde{R}_{\mathrm{r}}}{\beta^{2}} \tau_{\mathrm{d}} \beta \mathrm{e}^{J \rho_{\mathrm{d}}}\left[\begin{array}{l}
0 \\
1
\end{array}\right] .
$$

From this equation, $\tilde{x}(t)$ follows from the convolution integral

$$
\begin{aligned}
\tilde{x}(t)-\mathrm{e}^{-R_{\mathrm{r}} t} \tilde{x}(0) & =-\frac{1}{\beta} \int_{0}^{t} \mathrm{e}^{-R_{\mathrm{r}}(t-s)} \tilde{R}_{\mathrm{r}} \tau_{\mathrm{d}}(s) \mathrm{e}^{J \rho_{\mathrm{d}}(s)}\left[\begin{array}{l}
0 \\
1
\end{array}\right] \mathrm{d} s \\
& =-\frac{\tilde{R}_{\mathrm{r}} \beta}{\hat{R}_{\mathrm{r}}} \int_{0}^{t} \mathrm{e}^{-R_{\mathrm{r}}(t-s)} \dot{\rho}_{\mathrm{d}}(s) J \mathrm{e}^{J \rho_{\mathrm{d}}(s)} \mathrm{d} s\left[\begin{array}{l}
1 \\
0
\end{array}\right] \\
& =-\frac{\tilde{R}_{\mathrm{r}} \beta}{\hat{R}_{\mathrm{r}}} \int_{0}^{t} \mathrm{e}^{-R_{\mathrm{r}}(t-s)} \frac{\mathrm{de}^{J \rho_{\mathrm{d}}(s)}}{\mathrm{d} t} \mathrm{~d} s\left[\begin{array}{l}
1 \\
0
\end{array}\right] \\
& =-\frac{\tilde{R}_{\mathrm{r}} \beta}{\hat{R}_{\mathrm{r}}} \mathrm{e}^{-R_{\mathrm{r}} t} \int_{0}^{t} \mathrm{e}^{R_{\mathrm{r}}:} \frac{\mathrm{de}^{J \rho_{\mathrm{d}}(s)}}{\mathrm{d} t} \mathrm{~d} s\left[\begin{array}{l}
1 \\
0
\end{array}\right] .
\end{aligned}
$$

Integration by parts results in an expression for the integral

$$
\begin{aligned}
\int_{0}^{t} \mathrm{e}^{R_{\mathrm{r}} s} \frac{\mathrm{de}^{J \rho_{\mathrm{d}}(s)}}{\mathrm{d} s} \mathrm{~d} s & =\left.\mathrm{e}^{R_{\mathrm{r}} s} \mathrm{e}^{J \rho_{\mathrm{d}}}\right|_{0} ^{t}-\int_{0}^{t} \mathrm{e}^{J \rho_{\mathrm{d}}} R_{\mathrm{r}} \mathrm{e}^{R_{\mathrm{r}} s} \mathrm{~d} s \\
& =\mathrm{e}^{R_{\mathrm{r}} t} \mathrm{e}^{J \rho_{\mathrm{d}}(t)}-\mathrm{e}^{J \rho_{\mathrm{d}}(0)}-R_{\mathrm{r}} \int_{0}^{t} \mathrm{e}^{J \rho_{\mathrm{d}}} \mathrm{e}^{R_{\mathrm{r}} s} \mathrm{~d} s,
\end{aligned}
$$

so that, with initial conditions $x(0)=0, x_{\mathrm{d}}(0)=[\beta, 0]^{\mathrm{T}}$

$$
\begin{aligned}
& \mathrm{e}^{-J \rho_{\mathrm{d}}(t)} \tilde{x}(t)-\mathrm{e}^{-J \rho_{\mathrm{d}}(t)} \mathrm{e}^{-R_{\mathrm{r}} t} \tilde{x}(0) \\
& =-\frac{\tilde{R}_{\mathrm{r}} \beta}{\hat{R}_{\mathrm{\Gamma}}} \mathrm{e}^{-R_{\mathrm{r}} t} \mathrm{e}^{-J \rho_{\mathrm{d}}(t)}\left(\mathrm{e}^{R_{\mathrm{r}} t} \mathrm{e}^{J \rho_{\mathrm{d}}(t)}-\mathrm{e}^{J \rho_{\mathrm{d}}(0)}\right. \\
& \left.-R_{\mathrm{r}} \int_{0}^{1} \mathrm{e}^{J \rho_{\mathrm{d}}} \mathrm{e}^{R_{\mathrm{r}} s} \mathrm{~d} s\right)\left[\begin{array}{l}
1 \\
0
\end{array}\right] \\
& =-\frac{\tilde{R}_{\mathrm{r}} \beta}{\hat{R}_{\mathrm{r}}} \mathrm{e}^{-R_{\mathrm{r}} \mathrm{e}} \mathrm{e}^{-J\left(\rho_{\mathrm{d}}(t)-\rho_{\mathrm{d}}(0)\right)}\left[\begin{array}{l}
1 \\
0
\end{array}\right] \\
& =-\left(\mathrm{e}^{-R_{\mathrm{r}} t-J\left(\rho_{\mathrm{d}}(t)-\rho_{\mathrm{d}}(0)\right)}+1\right) \frac{\tilde{R}_{\mathrm{r}} \beta}{\hat{R}_{\mathrm{r}}}\left[\begin{array}{l}
1 \\
0
\end{array}\right] \\
& +\frac{\tilde{R}_{\mathrm{\Gamma}} \beta}{\hat{R}_{\mathrm{I}}} R_{\mathrm{\Gamma}} \int_{0}^{t} \mathrm{e}^{-R_{\mathrm{I}}(t-s)}\left[\begin{array}{c}
\cos z \\
-\sin z
\end{array}\right] \mathrm{d} s,
\end{aligned}
$$

where $z(t, \tau)$ is defined as

$$
z(t, s)=\rho_{\mathrm{d}}(t)-\rho_{\mathrm{d}}(s)
$$

\section{A.2. Elimination of $\tilde{x}$ from the expression for $\dot{\tau}_{\mathrm{d}}$} To eliminate $\mathrm{e}^{-J \rho_{\mathrm{d}}} \tilde{x}$ from the equation

$$
\dot{\tau}_{\mathrm{d}}=-\left(K_{P}+\frac{K_{I}}{p}\right)\left(\tau_{\mathrm{d}}-\tau_{L}-\left[-\frac{\tau_{\mathrm{d}}}{\beta} \beta\right] \mathrm{e}^{-J_{\mathrm{d}}} \tilde{x}\right)
$$

fill in

$$
\begin{aligned}
\mathrm{e}^{-J \rho_{\mathrm{f}} \tilde{x}=} & \mathrm{e}^{-R_{\mathrm{r}} t}\left[\begin{array}{l}
f_{1}(t) \\
f_{2}(t)
\end{array}\right]-\frac{\tilde{R}_{\mathrm{r}} \beta}{\hat{R}_{\mathrm{r}}}\left[\begin{array}{l}
1 \\
0
\end{array}\right] \\
& +\frac{\tilde{R}_{\mathrm{r}} \beta}{\hat{R}_{\mathrm{r}}} R_{\mathrm{r}} \int_{0}^{t} \mathrm{e}^{-R_{\mathrm{r}}(t-s)}\left[\begin{array}{c}
\cos z \\
-\sin z
\end{array}\right] \mathrm{d} s
\end{aligned}
$$

where functions $f_{1}, f_{2} \in \mathcal{L}_{\infty}$ depend on the initial values $\tilde{x}_{\mathrm{d}}(0), \rho_{\mathrm{d}}(0)$, so that

$$
\begin{aligned}
& {\left[-\frac{\tau_{\mathrm{d}}(t)}{\beta} \beta\right] \mathrm{e}^{-J \rho_{\mathrm{d}}} \tilde{x}=\mathrm{e}^{-R_{\mathrm{r}} t} f_{1}(t)} \\
& \quad+\tau_{\mathrm{d}} \mathrm{e}^{-R_{\mathrm{r}} t} f_{2}(t)+\frac{\tilde{R}_{\mathrm{r}}}{\hat{R}_{\mathrm{r}}} \tau_{\mathrm{d}}(t) \\
& \quad-\frac{\tilde{R}_{\mathrm{r}} \beta}{\hat{R}_{\mathrm{I}}} R_{\mathrm{r}} \int_{0}^{t} \mathrm{e}^{-R_{\mathrm{r}}(t-s)}\left(\frac{\tau_{\mathrm{d}}(t)}{\beta} \cos z+\beta \sin z\right) \mathrm{d} s,
\end{aligned}
$$

which finally leads to

$$
\begin{aligned}
\dot{\mathrm{T}}_{\mathrm{d}}=- & \left(K_{P}+\frac{K_{I}}{p}\right)\left(T_{\mathrm{d}}-\mathrm{T}_{L}+\mathrm{e}^{-R_{\mathrm{r}} t} f_{1}(t)+\tau_{\mathrm{d}} \mathrm{e}^{-R_{\mathrm{r}} t} f_{2}(t)\right. \\
& -\frac{\tilde{R}_{\mathrm{r}}}{\hat{R}_{\mathrm{r}}} T_{\mathrm{d}}(t) \\
& \left.+\frac{\tilde{R}_{\mathrm{r}} \beta}{\hat{R}_{\mathrm{r}}} R_{\mathrm{r}} \int_{0}^{t} \mathrm{e}^{-R_{\mathrm{r}}(t-s)}\left(\frac{\tau_{\mathrm{d}}(t)}{\beta} \cos z+\beta \sin z\right) \mathrm{d} s\right)
\end{aligned}
$$

With differential operator $p$, this can be also written as

$$
\begin{aligned}
& \left(p+\left(\frac{p K_{P}+K_{I}}{p}\right)\left(1-\frac{\tilde{R}_{\mathrm{r}}}{\hat{R}_{\mathrm{r}}}\right)\right) \tau_{\mathrm{d}}= \\
& \left(K_{P}+\frac{K_{I}}{p}\right)\left(\tau_{L}+\mathrm{e}^{-R_{\mathrm{r}} t} f_{\mathrm{l}}(t)+f_{2}(t) \mathrm{e}^{-R_{\mathrm{r}} t} \tau_{\mathrm{d}}\right) \\
& -\left(K_{P}+\frac{K_{I}}{p}\right) \frac{\tilde{R}_{\mathrm{r}} \beta}{\hat{R}_{\mathrm{r}}} R_{\mathrm{r}} \int_{0}^{t} \mathrm{e}^{-R_{\mathrm{r}}(t-s)}\left(\frac{\tau_{\mathrm{d}}(t)}{\beta} \cos (z)+\beta \sin z\right) \mathrm{d} s,
\end{aligned}
$$

so that $\tau_{\mathrm{d}}$ is the output of a linear time-invariant operator $G(p)$ with a nonlinear feedback $b(t)$

$$
\begin{aligned}
& \tau_{\mathrm{d}}(t)=\frac{p K_{P}+K_{I}^{2}}{p}+\left(p K_{P}+K_{I}\right)\left(1-\frac{\tilde{R}_{\mathrm{r}}}{\hat{R}_{\mathrm{r}}}\right) \\
& \times \frac{\tilde{R}_{\mathrm{r}} \beta R_{\mathrm{r}}}{\hat{R}_{\mathrm{r}}} \int_{0}^{t} \mathrm{e}^{-R_{\mathrm{r}}(t-s)}\left(\frac{\tau_{\mathrm{d}}(t)}{\beta} \cos z+\beta \sin z\right) \mathrm{d} s \\
& +\frac{p K_{P}+K_{I}}{p^{2}+\left(p K_{P}+K_{I}\right)\left(1-\frac{\tilde{R}_{\mathrm{r}}}{\hat{R}_{\mathrm{r}}}\right)} \\
& \times\left(\tau_{L}+f_{\mathrm{I}}(t) \mathrm{e}^{-R_{\mathrm{r}} r}+\tau_{\mathrm{d}} f_{2}(t) \mathrm{e}^{-R_{\mathrm{r}} r} .\right.
\end{aligned}
$$

\section{A.3. Feedback gain calculation}

The bounded signal $b(t)$ which is multiplied with $T_{\mathrm{d}}$ is

$$
h(t)=-\frac{\tilde{R}_{\mathrm{r}} R_{\mathrm{I}}}{\hat{R}_{\mathrm{r}}} \int_{0}^{t} \mathrm{e}^{-R_{\mathrm{r}}(t-s)} \cos z \mathrm{~d} s+f_{1}(t) \mathrm{e}^{-R_{\mathrm{r}} t},
$$

and it follows that $b(t)=b_{\infty}(t)+b_{1}(t)$ with

$$
\begin{gathered}
\left\|b_{\infty}(t)\right\|_{\infty}=\frac{\tilde{R}_{\mathrm{r}}}{\hat{R}_{\mathrm{r}}} \\
b_{1}(t) \in \mathcal{L}_{1} .
\end{gathered}
$$

